\title{
O “kit-covid" e o Programa Farmácia Popular do Brasil
}

\author{
"Kit-covid" and the Popular Pharmacy Program \\ in Brazil
}

\author{
"Kit-covid" y el Programa de Farmacia Popular \\ en Brasil
}

Cláudia Du Bocage Santos-Pinto 1
Elaine Silva Miranda 2
Claudia Garcia Serpa Osorio-de-Castro 3

doi: $10.1590 / 0102-311 \times 00348020$
A infecção pelo SARS-CoV-2 vem desafiando os países e seus sistemas de saúde, sendo a maior emergência de saúde pública de importância internacional já declarada 1 . O grande número de perdas de vidas se soma às dificuldades relacionadas ao manejo da doença, ainda sem tratamento eficaz disponível.

Desde o início da pandemia, muitos medicamentos já utilizados em outras doenças foram propostos como possibilidades terapêuticas contra COVID-19 - os chamados medicamentos "reposicionados”, entre eles a cloroquina e seu derivado, a hidroxicloroquina, a ivermectina, a nitazoxanida, o remdesivir e a azitromicina. Entretanto, quase um ano após o início da pandemia, não há evidências científicas que respaldem o uso dessas substâncias na prevenção ou tratamento da COVID-19 2,3,4,5,6.

Países que, em um primeiro momento, viram em alguns desses medicamentos uma esperança de cura, hoje os descartaram de seus protocolos 7. Em junho de 2020, o governo dos Estados Unidos suspendeu a autorização de uso emergencial que permitia que o fosfato de cloroquina e o sulfato de hidroxicloroquina fossem utilizados para tratar pacientes hospitalizados com COVID-19, fora de ensaios clínicos. O comunicado emitido pela Agência Americana de Administração de Alimentos e Medicamentos (FDA), dizia que os eventuais benefícios atribuídos à cloroquina e hidroxicloroquina não compensavam os riscos conhecidos de seu uso 8 .

Mesmo diante deste cenário, a postura adotada pelo governo brasileiro foi a de incentivar a utilização dos medicamentos, dando à cloroquina e à hidroxicloroquina status de "bala de prata" contra a COVID-19. Protocolos do Ministério da Saúde, ainda vigentes, incluem estes medicamentos como indicações para o manejo de pacientes com sintomas leves, moderados e graves, e nas diversas fases de evolução da doença 9 .

A promoção do uso de cloroquina e hidroxicloroquina trouxe consequências diversas. Uma pesquisa do Conselho Federal de Farmácia (CFF) comparou as vendas de medicamentos e suplementos alimentares, de janeiro a março de 2019, com as de 2020, mostrando um aumento de $68 \%$ nas vendas de hidroxicloroquina nesse período 10 . Em decorrência, foi observada escassez generalizada do medicamento nas farmácias, prejudicando pacientes que dele dependiam para outras condições de saúde 11.

No final de maio de 2020, o Governo Federal anunciou o recebimento de uma doação dos Estados Unidos de cerca de 2 milhões de doses de hidroxicloroquina 12,13. O momento coincidiu com o anúncio do abandono do uso desse medicamento para tratamento da COVID-19 nos Estados Unidos. A doação foi recebida pelo Laboratório Químico e Farmacêutico do Exército, e se somou ao quantitativo
${ }^{1}$ Instituto Integrado de Saúde, Universidade Federal de Mato Grosso do Sul, Campo Grande, Brasil. 2 Faculdade de Farmácia, Universidade Federal Fluminense, Niterói, Brasil. ${ }^{3}$ Escola Nacional de Saúde Pública Sergio Arouca, Fundação Oswaldo Cruz, Rio de Janeiro, Brasil.

\section{Correspondência} C. D. B. Santos-Pinto Instituto Integrado de Saúde, Universidade Federal de Mato Grosso do Sul. Av. Costa e Silva s/n, Campo Grande, MS 79070-900, Brasil.

bocage.santos@ufms.br 
já produzido pelo órgão ${ }^{14}$. Esse investimento do governo brasileiro na produção de cloroquina se tornou alvo de investigação por parte do Tribunal de Contas da União (TCU) 15, e também de questionamento pela Câmara dos Deputados, em função do montante gasto na produção de um medicamento sem comprovação científica para COVID-19, o que poderia ferir o princípio da eficiência na administração pública 16 .

Relatório que analisou padrões de desinformação entre países durante a pandemia mostrou, por meio de análises hipergeométrica e qualitativa, que o Brasil é o país que mais se destaca no que diz respeito à desinformação relacionada a medicamentos. Cloroquina e hidroxicloroquina continuam sendo mencionados como possíveis tratamentos ao longo da pandemia, indicando que as evidências científicas não estão sendo adequadamente captadas pelo debate público brasileiro 17 . Tal fato tem forte relação com o posicionamento de autoridades e órgãos governamentais, como o próprio Ministério da Saúde, mostrando a desinformação como tática intimamente ligada às disputas políticas internas no país.

A defesa do "tratamento precoce", baseado em cloroquina/hidroxicloroquina e outros medicamentos, tornou-se, no Brasil, símbolo do viés político no enfrentamento da epidemia. A alternativa dada pelo governo perpassa a disponibilização do dito "kit-covid" em unidades básicas de saúde, o que foi adotado em alguns municípios brasileiros, alinhados com a premissa do Governo Federal 18 . O "kit-covid" consiste em uma variação de combinações que incluem, invariavelmente, a cloroquina/ hidroxicloroquina, a azitromicina, a ivermectina, e mais outros medicamentos, a depender da localidade. Agora, o Governo Federal sinaliza para a disponibilização do "kit-covid” também nas farmácias conveniadas do Programa Farmácia Popular do Brasil (PFPB) 19.

O PFPB foi criado com o intuito de ampliar o acesso a medicamentos no país, visando as condições de saúde mais prevalentes, e a parcela da população com menos recursos. Estima-se que, durante muito tempo, guardadas as críticas existentes sobre o direcionamento de recursos para o setor privado em detrimento do investimento público, o PFPB foi capaz de cumprir seu propósito, seguindo os preceitos dispostos na Política Nacional de Assistência Farmacêutica, e na Política Nacional de Medicamentos. Observou-se um grande volume de usuários atendidos e uma grande capilaridade do PFPB, por meio das unidades próprias e conveniadas em todo país 20,21.

No primeiro momento da pandemia de COVID-19, o PFPB, coadunante com as regras de distanciamento social, se organizou para ampliar as quantidades de medicamentos do Programa autorizadas para dispensação, por paciente, e facilitou sua retirada por terceiros, visando diminuir a circulação de pessoas, principalmente aquelas pertencentes a grupo de risco 22 . No entanto, em dezembro de 2020 , o PFPB passa a ser noticiado pela possibilidade de dispensação do "kit-covid" 19.

Esta não é a primeira vez que o PFPB desempenha papel durante emergências sanitárias. O fosfato de oseltamivir (Tamiflu), utilizado no tratamento da gripe H1N1, começou a ser dispensado pelo PFPB em abril de 2010 23. O Instituto de Tecnologia em Fármacos da Fundação Oswaldo Cruz (Farmanguinhos/Fiocruz) foi o responsável pela produção do medicamento, a partir do princípio ativo que o Ministério da Saúde tinha em estoque, e todas as unidades próprias do PFPB - cerca de 530 à época - passaram a disponibilizar, no total, mais de 2 milhões de tratamentos a pacientes com sintomas de gripe 24 . O oseltamivir também foi disponibilizado nas farmácias privadas conveniadas, com subsídios do governo 23. O Ministério da Saúde recomendou também às Secretarias de Saúde dos estados e municípios que descentralizassem os estoques do medicamento, de modo a proporcionar o acesso imediato 25 . Ressalta-se que a indicação do medicamento então aprovada no país, dispunha que o oseltamivir deveria ser utilizado apenas em pacientes com quadro de doença respiratória grave, e nos quais o início dos sintomas tivesse ocorrido nas 48 horas anteriores.

Naquele momento havia início de pânico instaurado e, ao mesmo tempo, a propaganda massiva do produto pela indústria. Deste modo, o oseltamivir, que posteriormente foi reconhecido pela comunidade científica como tendo eficácia modesta e limitada no combate ao H1N1, foi transformado em objeto de desejo de pessoas com quaisquer sintomas suspeitos 26 , aumentando o volume de prescrições. A dispensação nas unidades da PFPB por todo o país, patrocinada pelo Governo Federal, oportunizou a discussão sobre a qualidade desse uso e a apropriação do Programa para distribuição dos estoques do medicamento. 
Diante da pandemia da COVID-19, novamente aventa-se o uso da Farmácia Popular para promover a distribuição de medicamentos. Desta vez, porém, além de não existirem evidências de benefícios de eficácia e segurança na doença em questão, há claras indicações de potenciais riscos. $\mathrm{O}$ uso de cloroquina e seus derivados pode levar ao agravamento de quadros de pacientes com doença cardíaca prévia 27. Somam-se assim novas questões, envolvendo a prescrição e a dispensação de medicamentos para uso não aprovado pelo órgão regulador, amplificando os riscos sanitários relacionados à pandemia.

O uso off-label é, por definição, não autorizado pela agência reguladora, mas, muitas vezes, é um uso absolutamente necessário, como quando os trâmites de autorização não avançam na mesma velocidade de evidências de benefício, ou quando a falta de ensaios clínicos em alguns grupos de pacientes impede a indicação que se estima segura e potencialmente eficaz nesses grupos 28 .

Admitiu-se o uso off-label para a cloroquina e para a hidroxicloroquina no momento inicial da pandemia, quando ainda pairavam as hipóteses sobre sua eficácia na COVID-19. O Conselho Federal de Medicina (CFM) se posicionou apontando que o uso prescrito desses medicamentos na COVID-19 poderia ser resultante de consenso entre médico e paciente 29. Entretanto, esta posição encontra-se seriamente abalada após a publicação de consensos de especialistas no Brasil 30,31,32,33 e de estudos que apontam a falta de beneficio clínico destes medicamentos 3,4,5, aumentando o questionamento da persistência das prescrições e da dispensação destes medicamentos para a COVID-19.

A Assistência Farmacêutica, parte integrante e essencial do sistema de saúde brasileiro, é responsável pela disponibilização de medicamentos à população, prezando pelos princípios de segurança e eficácia, e tendo o uso racional sempre como norte de suas atividades. Em um cenário de pandemia, com incertezas que cercam o uso de medicamentos, a orientação farmacêutica se faz mais necessária do que nunca. O PFPB hoje, diferente do momento vivenciado pela epidemia de H1N1, está exclusivamente concentrado no comércio varejista de medicamento. Nas farmácias privadas, a dispensação por vezes é executada por indivíduos sem capacitação técnica ou obrigação de prestar esclarecimentos e orientar sobre o uso racional de medicamentos (URM) 34 . Deste modo, quais seriam os resultados esperados da oferta não monitorada de medicamentos do "kit-covid" pelo PFPB?

A possibilidade de o governo brasileiro usar a PFPB como repositório e distribuidor de medicamentos desprovidos de evidência de eficácia e segurança poderá causar, primeiramente, o desperdício de recursos públicos direcionados para a distribuição dos medicamentos e para ressarcimento dos possíveis custos operacionais das farmácias privadas. Recursos esses que poderiam ser redirecionados para atividades efetivas de resposta à pandemia. E em segundo lugar, o ponto mais preocupante: a exposição da população brasileira a riscos inadmissíveis, potencialmente relacionados ao uso não racional destes medicamentos.

Assistimos, assim, à subversão do papel do PFPB pelo governo brasileiro, que parece colocar a agenda política à frente das prioridades sanitárias e do bem-estar da população. 


\section{Colaboradores}

C. D. B. Santos-Pinto contribuiu com a concepção, redação do artigo, revisão crítica e aprovação da versão final. E. S. Miranda e C. G. S. Osorio-de-Castro contribuíram com a redação do artigo, revisão crítica, aprovação da versão final.

\section{Informações adicionais}

ORCID: Cláudia Du Bocage Santos-Pinto (00000002-5478-4977); Elaine Silva Miranda (00000002-6204-5023); Claudia Garcia Serpa Osorio-deCastro (0000-0003-4875-7216).

\section{Referências}

1. Gostin LO. The great coronavirus pandemic of 2020 - 7 critical lessons. JAMA 2020; 324:1816-7.

2. Beigel JH, Tomashek KM, Dodd LE, Mehta AK, Zingman BS, Kalil AC, et al. Remdesivir for the treatment of Covid-19 - final report. N Engl J Med 2020; 383:1813-26.

3. Cavalcanti AB, Zampieri FG, Rosa RG, Azevedo LCP, Veiga VC, Avezum A, et al. Hydroxychloroquine with or without azithromycin in mild-to-moderate Covid-19. N Engl J Med 2020; 383:2041-52.

4. Horby P, Mafham M, Linsell L, Bell JL, Staplin $\mathrm{N}$, Emberson JR, et al. Effect of hydroxychloroquine in hospitalized patients with Covid-19. N Engl J Med 2020; 383:2030-40.

5. Mitjà $\mathrm{O}$, Corbacho-Monné $\mathrm{M}$, Ubals $\mathrm{M}$, Alemany A, Suñer C, Tebé C, et al. A cluster-randomized trial of hydroxychloroquine for prevention of Covid-19. N Engl J Med 2020; (Online ahead of print).

6. Siemieniuk RAC, Bartoszko JJ, Ge L, Zeraatkar D, Izcovich A, Kum E, et al. Drug treatments for covid-19 living systematic review and metanalysis. BMJ 2020; 370:m2980.

7. Centers for Disease Control and Prevention. Interim clinical guidance for management of patients with confirmed coronavirus disease (COVID-19). https://www.cdc.gov/ coronavirus/2019-ncov/hcp/clinical-guid ance-management-patients.html (acessado em 14/Dez/2020).

8. U.S. Food and Drug Administration. Coronavirus (COVID-19) update: FDA revokes emergency use authorization for chloroquine and hydroxychloroquine. https://www.fda.gov/ news-events/press-announcements/coronavi rus-covid-19-update-fda-revokes-emergencyuse-authorization-chloroquine-and (acessado em 12/Dez/2020).

9. Ministério da Saúde. Nota Informativa no 17/2020- SE/GAB/SE/MS. Orientações do Ministério da Saúde para manuseio medicamentoso precoce de pacientes com diagnóstico da Covid-19. http://antigo.saude.gov.br/ images/pdf/2020/August/12/COVID-11ago 2020-17h16.pdf (acessado em 12/Dez/2020).

10. Conselho Federal de Farmácia. Levantamento mostra como o medo da Covid-19 impactou venda de medicamentos. https://www.cff. org.br/noticia.php?id=5747 (acessado em 12/ Dez/2020).
11. Conselho Nacional de Saúde. CNS recomenda ao Ministério da Saúde plano de abastecimento de cloroquina para pacientes com doenças crônicas e patologias. http://conselho.saude.gov. br/ultimas-noticias-cns/1323-cns-recomen da-ao-ministerio-da-saude-plano-de-abaste cimento-de-cloroquina-para-pacientes-comdoencas-cronicas-e-patologias (acessado em 12/Dez/2020).

12. Verdélio A. Brasil recebe 2 milhões de doses de hidroxicloroquina dos EUA. Agência Brasil 2002; 1 jun. https://agenciabrasil.ebc.com.br/ politica/noticia/2020-06/brasil-recebe-doismilhoes-de-doses-de-hidroxicloroquina-doseua.

13. Ministério das Relações Exteriores. Nota à imprensa no 62. Declaração conjunta dos governos da República Federativa do Brasil e dos Estados Unidos da América relativa à cooperação em saúde. https://www.gov.br/mre/pt-br/canais_ atendimento/imprensa/notas-a-imprensa/ declaracao-conjunta-dos-governos-da-republi ca-federativa-do-brasil-e-dos-estados-unidosda-america-relativa-a-cooperacao-em-saude-2 (acessado em 12/Dez/2020).

14. Exército Brasileiro. Laboratório químico farmacêutico do exército intensifica a produção de cloroquina. https://www.eb.mil.br/web/noti cias/noticiario-do-exercito/-/asset_publisher/ MjaG93KcunQI/content/id/11267194 (acessado em 12/Dez/2020).

15. Tribunal de Contas da União. Processo 039.440/2020-6. https://pesquisa.apps. tcu.gov.br/\#/push/processo? numero= 039.440\%2F2020-6 (acessado em 12/Dez/ 2020).

16. Câmara dos Deputados. Requerimento de informação no 1.194, de 2020. Diário da Câmara dos Deputados. http://imagem.camara.gov.br/ Imagem/d/pdf/DCD0020200925001680000. PDF\#page $=($ acessado em 12/Dez/2020 $)$.

17. Machado CCV, Santos JG, Santos N, Bandeira L. International trends in misinformation and the departure from the scientific debate. https://laut.org.br/wp-content/up loads/2020/11/Political-Self-Isolation-vF.pdf (acessado em 12/Dez/2020). 
18. Celestino C. Centro de Triagem entregou mais de 12 mil kits de medicamentos para tratamento da Covid-19. Governo do Mato Grosso - Notícias 2020; 2 set. http://www.mt.gov. $\mathrm{br} /$-/15304126-centro-de-triagem-entregoumais-de-12-mil-kits-de-medicamentos-paratratamento-da-covid-19.

19. Vargas M. Saúde prevê gastar R\$ 250 milhões para distribuir "kit covid". O Estado de S.Paulo 2002; $11 \mathrm{dez}$. https://saude.estadao.com.br/ noticias/geral,saude-preve-gastar-r-250-mi lhoes-para-por-kit-covid-em-farmacias-popu lares, 70003547892.

20. Santos-Pinto CDB, Costa NR, Osório-de-Castro CGS. Quem acessa o Programa Farmácia Popular do Brasil? Aspectos do fornecimento público de medicamentos. Ciênc Saúde Colet 2011; 16:2963-73.

21. Silva RM, Caetano R. Programa "Farmácia Popular do Brasil”: caracterização e evolução entre 2004-2012. Ciênc Saúde Colet 2015; 20:2943-56.

22. Ministério da Saúde. Alterações no programa farmácia popular devido à situação de emergência de saúde pública decorrente do coronavírus (covid19). https://antigo.saude.gov.br/ noticias/assistencia-farmaceutica/46583-alte racoes-no-programa-farmacia-popular-devido-a-situacao-de-emergencia-de-saude-publicadecorrente-do-coronavirus-covid19 (acessado em 14/Dez/2020).

23. Ministério da Saúde. Portaria no 367, de 22 de fevereiro de 2010. Inclui o medicamento Fosfato de Oseltamivir no Programa Farmácia Popular do Brasil - Aqui Tem Farmácia Popular, e define os valores de referência para as suas apresentações. Diário Oficial da União 2010; 23 fev.

24. Fundação Oswaldo Cruz. Farmácia Popular oferece remédio contra gripe H1N1. https:// portal.fiocruz.br/noticia/farmacia-popularoferece-remedio-contra-gripe-h1n1 (acessado em 14/Dez/2020).

25. Organização Pan-Americana da Saúde. Ministério da Saúde informa que a utilização oportuna do oseltamivir reduz risco de óbito por influenza A (H1N1). https://www.paho.org/bra/in dex.php?option=com_content\&view $=$ article \& $\mathrm{id}=3455$ :ministerio-da-saude-informa-que-autilizacao-oportuna-do-oseltamivir-reduzrisco-de-obito-por-influenza-a-h1n1-3\& Itemid $=812$ (acessado em 14/Dez/2020).
26. Gupta YK, Meenu M, Mohan P. The Tamiflu fiasco and lessons learnt. Indian J Pharmacol 2015; 47:11-6.

27. Borba MGS, Val FFA, Sampaio VS, Alexandre MAA, Melo GC, Brito M, et al. Effect of high vs low doses of chloroquine diphosphate as adjunctive therapy for patients hospitalized with severe acute respiratory syndrome coronavirus 2 (SARS-CoV-2) infection: a randomized clinical trial. JAMA Netw Open 2020; 3:e208857.

28. Paumgartten FJR, Oliveira ACAX. Uso off label, compassivo e irracional de medicamentos na pandemia de Covid-19, consequências para a saúde e questões éticas. Ciênc Saúde Coletiva 2020; 25:3413-9.

29. Conselho Federal de Medicina. Parecer CFM no 4/2020. https://sistemas.cfm.org.br/nor mas/visualizar/pareceres/BR/2020/4 (acessado em 16/Dez/2020).

30. Sociedade Brasileira de Infectologia. Atualizações e recomendações sobre a COVID-19. https://infectologia.org.br/wp-content/ uploads/2020/12/atualizacoes-e-recomenda coes-covid-19.pdf (acessado em 16/Dez/2020).

31. Associação de Medicina Intensiva Brasileira. Diretrizes para o tratamento farmacológico da COVID-19. https://www.amib.org.br/filead $\mathrm{min} /$ user_upload/amib/2020/maio/19/Dire trizes_para_o_Tratamento_Farmacologico_ da_COVID_-_v18mai2020__2_.pdf (acessado em 16/Dez/2020).

32. Falavigna M, Colpani V, Stein C, Azevedo LC, Bagattini AM, Brito GV, et al. Diretrizes para o tratamento farmacológico da COVID-19. Rev Bras Ter Intensiva 2020; 32:166-96.

33. National Institutes of Health. Coronavirus disease 2019 (COVID-19) treatment guidelines. https://www.covid19treatmentguidelines.nih. gov/whats-new/ (acessado em 16/Dez/2020).

34. Conselho Federal de Farmácia. Dados 2019. https://www.cff.org.br/pagina. php?id=801\&titulo=Dados +2018 (acessado em 16/Dez/2020). 